\title{
Publisher Correction: Regioselective surface encoding of nanoparticles for programmable self-assembly
}

Gang Chen, Kyle J. Gibson, Di Liu, Huw C. Rees, Jung-Hoon Lee, Weiwei Xia, Ruoqian Lin, Huolin L. Xin (D), Oleg Gang (iD and Yossi Weizmann (D)

Correction to: Nature Materials https://doi.org/10.1038/s41563-018-0231-1 published online 3 December 2018.

In the version of this Article originally published, the diblock copolymer structure in Fig. 2a showed a single bond between the carbon and the oxygen atoms; it should have been a double bond. This has been corrected in all versions of the Article.

Published online: 12 December 2018

https://doi.org/10.1038/s41563-018-0269-0 